\title{
HOMOGENOUS BANACH SPACES ON THE UNIT CIRCLE
}

\author{
Thomas Vils Pedersen*
}

\begin{abstract}
We prove that a homogeneous Banach space $\mathcal{B}$ on the unit circle $\mathbb{T}$ can be embedded as a closed subspace of a dual space $\Xi_{\mathcal{B}}^{*}$ contained in the space of bounded Borel measures on $\mathbb{T}$ in such a way that the map $\mathcal{B} \mapsto \Xi_{\mathcal{B}}^{*}$ defines a bijective correspondence between the class of homogeneous Banach spaces on $\mathbb{T}$ and the class of prehomogeneous Banach spaces on $\mathbb{T}$.

We apply our results to show that the algebra of all continuous functions on $\mathbb{T}$ is the only homogeneous Banach algebra on $\mathbb{T}$ in which every closed ideal has a bounded approximate identity with a common bound, and that the space of multipliers between two homogeneous Banach spaces is a dual space. Finally, we describe the space $\Xi_{\mathcal{B}}^{*}$ for some examples of homogeneous Banach spaces $\mathcal{B}$ on $\mathbb{T}$.
\end{abstract}

\section{Introduction}

Every Banach space $\mathcal{Z}$ is a closed subspace of its second dual space $\mathcal{Z}^{* *}$, but the restriction of the weak star ( $\left.\mathrm{wk}^{*}\right)$ topology on $\mathcal{Z}^{* *}$ to $\mathcal{Z}$ (which is of course just the weak topology on $\mathcal{Z}$ ) need not be particularly useful. Loosely speaking, this is because the space $\mathcal{Z}^{* *}$ is too large and we are therefore interested in embedding $\mathcal{Z}$ in a smaller dual space. In this paper, we show that a Banach space $\mathcal{B}$ of functions on the unit circle $\mathbb{T}$ satisfying certain conditions can be embedded in a dual space contained in the space $\mathcal{M}$ of bounded Borel measures on $\mathbb{T}$ in such a way, that convergence of a sequence in $\mathcal{B}$ in the wk ${ }^{*}$ topology depends only on certain simple properties of the sequence. For some Banach spaces $\mathcal{B}$ of functions on $\mathbb{T}$, there is a "natural" dual space in which $\mathcal{B}$ is embedded. Examples of such embeddings are that of $L^{1}$ in $\mathcal{M}$, of $\mathcal{C}$ (the algebra

1991 Mathematics Subject Classification. 46E30, 46E15, 46J10.

* Supported by the Danish Natural Science Research Council and by a TMR Marie

Curie postdoctoral grant from the European Commission. 
of all continuous functions on $\mathbb{T}$ ) in $L^{\infty}$ and of the little Lipschitz algebra $\lambda_{\gamma}$ in the big Lipschitz algebra $\Lambda_{\gamma}$. We shall see that our general approach reproduces these examples. We wish to thank the referee for pointing out a mistake in our original formulation of Theorem 4.3.

Following Shilov $([\mathbf{2 0}]$, see also $[\mathbf{1 3}])$, we say that a Banach space $\mathcal{B}$ continuously embedded in $L^{1}$ is a homogeneous Banach space on $\mathbb{T}$ if it satisfies the following condition. For $f \in \mathcal{B}$ and $s \in \mathbb{T}$, the translate

$$
f_{s}(t)=f(t-s) \quad(t \in \mathbb{T})
$$

belongs to $\mathcal{B}$ with $\left\|f_{s}\right\|_{\mathcal{B}}=\|f\|_{\mathcal{B}}$ and $f_{s} \rightarrow f$ in $\mathcal{B}$ as $s \rightarrow 0$. For $s \in \mathbb{T}$, the translation operator $f \mapsto f_{s}(f \in \mathcal{B})$ is denoted by $R_{s}$.

For $f \in L^{1}$, let $\widehat{f}(n)(n \in \mathbb{Z})$ be the Fourier coefficients of $f$ and, for $N \in \mathbb{N}$, let

$$
\sigma_{N}(f)=\sum_{n=-N}^{N}\left(1-\frac{|n|}{N+1}\right) \widehat{f}(n) \alpha^{n}
$$

be the $N$ 'th Fejér sum of $f$ (where $\alpha^{n}(t)=e^{\text {int }}$ for $t \in \mathbb{T}$ ). The following result ([20, pp. 12-13] or [13, Theorem I.2.11]) is essential for our approach.

Theorem 1.1 (Shilov). Let $\mathcal{B}$ be a homogeneous Banach space on $\mathbb{T}$ and let $f \in \mathcal{B}$. If $\widehat{f}(n) \neq 0$ for some $n \in \mathbb{Z}$, then $\alpha^{n} \in \mathcal{B}$. Moreover,

$$
\sigma_{N}(f) \rightarrow f
$$

in $\mathcal{B}$ as $N \rightarrow \infty$ and $\left\|\sigma_{N}(f)\right\|_{\mathcal{B}} \leq\|f\|_{\mathcal{B}}$ for $N \in \mathbb{N}$. In particular, the trigonometric polynomials in $\mathcal{B}$ are dense in $\mathcal{B}$.

For $n \in \mathbb{Z}$, define $\xi_{n} \in \mathcal{B}^{*}$ by

$$
\left\langle f, \xi_{n}\right\rangle=\widehat{f}(n) \quad(f \in \mathcal{B}) .
$$

In passing, we remark that the definition of a homogeneous Banach space $\mathcal{B}$ on $\mathbb{T}$ does not exclude the case where $\widehat{f}(n)=0$ for every $f \in \mathcal{B}$ (that is $\xi_{n}=0$ ) for some values of $n$. In particular, certain spaces of analytic functions are homogeneous Banach spaces on $\mathbb{T}$. (See Section 5 for further details.) For $\varphi \in \mathcal{B}^{*}$ and $n \in \mathbb{Z}$, let

$$
\widehat{\varphi}(n)=\left\langle\alpha^{n}, \varphi\right\rangle .
$$

Since the trigonometric polynomials in $\mathcal{B}$ are dense in $\mathcal{B}$, the map $\varphi \mapsto$ $(\widehat{\varphi}(n))$ is $1-1$ on $\mathcal{B}^{*}$. 
In this paper, the subspace

$$
\left.\Xi_{\mathcal{B}}=\overline{\operatorname{span}\left\{\xi_{n}: n \in \mathbb{Z}\right.}\right\}^{\mathcal{B}^{*}}
$$

of $\mathcal{B}^{*}$ plays an important part. For $\varphi \in \mathcal{B}^{*}$, define the Fejér sums of $\varphi$ by

$$
\sigma_{N}(\varphi)=\sum_{n=-N}^{N}\left(1-\frac{|n|}{N+1}\right) \widehat{\varphi}(n) \xi_{n} \quad(N \in \mathbb{N})
$$

and, for $s \in \mathbb{T}$, define the translate $\varphi_{s} \in \mathcal{B}^{*}$ by $\left\langle f, \varphi_{s}\right\rangle=\left\langle f_{s}, \varphi\right\rangle(f \in \mathcal{B})$. Since $\left(\xi_{n}\right)_{s}=e^{-i n s} \xi_{n}(s \in \mathbb{T}, n \in \mathbb{Z})$, it follows that $\Xi_{\mathcal{B}}$ is translation invariant. The previous theorem leads to the following result in the dual space.

Proposition 1.2. Let $\mathcal{B}$ be a homogeneous Banach space on $\mathbb{T}$ and let $\varphi \in \mathcal{B}^{*}$. Then $\sigma_{N}(\varphi) \rightarrow \varphi \mathrm{wk}^{*}$ in $\mathcal{B}^{*}$ as $N \rightarrow \infty$ and $\left\|\sigma_{N}(\varphi)\right\|_{\mathcal{B}^{*}} \leq$ $\|\varphi\|_{\mathcal{B}^{*}}$ for $N \in \mathbb{N}$. In particular, the unit ball of $\Xi_{\mathcal{B}}$ is $\mathrm{wk}^{*}$ sequentially dense in the unit ball of $\mathcal{B}^{*}$. Moreover, $\varphi_{s} \rightarrow \varphi \mathrm{wk}^{*}$ in $\mathcal{B}^{*}$ as $s \rightarrow 0$. For $\varphi \in \Xi_{\mathcal{B}}$, we have $\sigma_{N}(\varphi) \rightarrow \varphi$ in $\Xi_{\mathcal{B}}$ as $N \rightarrow \infty$ and $\varphi_{s} \rightarrow \varphi$ in $\Xi_{\mathcal{B}}$ as $s \rightarrow 0$.

Proof: For $f \in \mathcal{B}$ and $N \in \mathbb{N}$, we have

$$
\left\langle f, \sigma_{N}(\varphi)\right\rangle=\sum_{n=-N}^{N}\left(1-\frac{|n|}{N+1}\right) \widehat{f}(n) \widehat{\varphi}(n)=\left\langle\sigma_{N}(f), \varphi\right\rangle,
$$

so the first two conclusions follows from the previous theorem. Also, the maps $\varphi \mapsto \sigma_{N}(\varphi)$ define a bounded sequence of operators on $\Xi_{\mathcal{B}}$, and since $\sigma_{N}\left(\xi_{m}\right) \rightarrow \xi_{m}$ in $\Xi_{\mathcal{B}}$ as $N \rightarrow \infty$ for $m \in \mathbb{Z}$, it follows that $\sigma_{N}(\varphi) \rightarrow \varphi$ in $\Xi_{\mathcal{B}}$ for $\varphi \in \Xi_{\mathcal{B}}$. The results about $\varphi_{s}$ follow in the same way.

\section{Embedding a homogeneous Banach space in a dual space}

We shall now focus on the space $\Xi_{\mathcal{B}}^{*}$. For $\mu \in \Xi_{\mathcal{B}}^{*}$ and $n \in \mathbb{Z}$, let

$$
\widehat{\mu}(n)=\left\langle\xi_{n}, \mu\right\rangle .
$$

By definition of $\Xi_{\mathcal{B}}$, it follows that the map $\mu \mapsto(\widehat{\mu}(n))$ is $1-1$ on $\Xi_{\mathcal{B}}^{*}$. The following is the key result of this paper. 
Theorem 2.1. Let $\mathcal{B}$ be a homogeneous Banach space on $\mathbb{T}$ and define

$$
\Phi: \mathcal{B} \rightarrow \Xi_{\mathcal{B}}^{*}
$$

by

$$
\langle\varphi, \Phi(f)\rangle=\langle f, \varphi\rangle
$$

for $f \in \mathcal{B}$ and $\varphi \in \Xi_{\mathcal{B}}$. (That is, $\Phi(f)=\left.\iota(f)\right|_{\Xi_{\mathcal{B}}}(f \in \mathcal{B})$, where $\iota: \mathcal{B} \rightarrow \mathcal{B}^{* *}$ is the canonical embedding.) Then $\Phi$ is an isometry and

$$
\widehat{\Phi(f)}(n)=\widehat{f}(n)
$$

for $f \in \mathcal{B}$ and $n \in \mathbb{Z}$. Moreover, a sequence $\left(\mu_{m}\right)$ in $\Xi_{\mathcal{B}}^{*}$ converges $\mathrm{wk}^{*}$ to 0 in $\Xi_{\mathcal{B}}^{*}$ as $m \rightarrow \infty$ if and only if the sequence is bounded in $\Xi_{\mathcal{B}}^{*}$ and $\widehat{\mu_{m}}(n) \rightarrow 0$ as $m \rightarrow \infty$ for every $n \in \mathbb{Z}$.

Proof: Let $f \in \mathcal{B}$. It follows from Proposition 1.2 that

$$
\|f\|_{\mathcal{B}}=\sup \left\{|\langle f, \varphi\rangle|: \varphi \in \Xi_{\mathcal{B}},\|\varphi\|_{\mathcal{B}^{*}} \leq 1\right\}=\|\Phi(f)\|_{\Xi_{\mathcal{B}}^{*}}
$$

Also,

$$
\widehat{\Phi(f)}(n)=\left\langle\xi_{n}, \Phi(f)\right\rangle=\left\langle f, \xi_{n}\right\rangle=\widehat{f}(n) \quad(n \in \mathbb{Z}),
$$

so $\widehat{\Phi(f)}=\widehat{f}$. The last statement follows immediately, since $\operatorname{span}\left\{\xi_{n}\right.$ : $n \in \mathbb{Z}\}$ is dense in $\Xi_{\mathcal{B}}$.

Because of the theorem, we shall from now on consider $\mathcal{B}$ as a closed subspace of $\Xi_{\mathcal{B}}^{*}$ and identify $\Phi(f)$ and $f$ for $f \in \mathcal{B}$. We shall use the following simple result several times.

Lemma 2.2. Let $\mathcal{B}$ be a homogeneous Banach space on $\mathbb{T}$ and suppose that $\widehat{\mu}(n) \neq 0$ for some $\mu \in \Xi_{\mathcal{B}}^{*}$ and $n \in \mathbb{Z}$. Then $\alpha^{n} \in \mathcal{B}$. In particular, if $p \in \Xi_{\mathcal{B}}^{*}$ is a trigonometric polynomial, then $p \in \mathcal{B}$.

Proof: Since $\left\langle\xi_{n}, \mu\right\rangle=\widehat{\mu}(n) \neq 0$, we have $\xi_{n} \neq 0$ in $\Xi_{\mathcal{B}} \subseteq \mathcal{B}^{*}$ and thus $\widehat{f}(n) \neq 0$ for some $f \in \mathcal{B}$. Hence $\alpha^{n} \in \mathcal{B}$ by Theorem 1.1. The last statement is now obvious.

For $\mu \in \Xi_{\mathcal{B}}^{*}$ and $N \in \mathbb{N}$, let

$$
\sigma_{N}(\mu)=\sum_{n=-N}^{N}\left(1-\frac{|n|}{N+1}\right) \widehat{\mu}(n) \alpha^{n}
$$

be the Fejér sums of $\mu$. Then $\sigma_{N}(\mu) \in \mathcal{B}$ by the previous lemma.

Corollary 2.3. Let $\mathcal{B}$ be a homogeneous Banach space on $\mathbb{T}$. For $\mu \in$ $\Xi_{\mathcal{B}}^{*}$, we have

$$
\sigma_{N}(\mu) \rightarrow \mu \quad \mathrm{wk}^{*}
$$

in $\Xi_{\mathcal{B}}^{*}$ as $N \rightarrow \infty$ and $\left\|\sigma_{N}(\mu)\right\|_{\mathcal{B}} \leq\|\mu\|_{\Xi_{\mathcal{B}}^{*}}$ for $N \in \mathbb{N}$. 
Proof: For $\varphi \in \Xi_{\mathcal{B}}$ and $N \in \mathbb{N}$, we have

$$
\left\langle\varphi, \sigma_{N}(\mu)\right\rangle=\sum_{n=-N}^{N}\left(1-\frac{|n|}{N+1}\right) \widehat{\varphi}(n) \widehat{\mu}(n)=\left\langle\sigma_{N}(\varphi), \mu\right\rangle .
$$

Since $\left\|\sigma_{N}(\varphi)\right\|_{\Xi_{\mathcal{B}}} \leq\|\varphi\|_{\Xi_{\mathcal{B}}}$ by Proposition 1.2, it follows that $\left\|\sigma_{N}(\mu)\right\|_{\mathcal{B}} \leq$ $\|\mu\|_{\Xi_{\mathcal{B}}^{*}}$. Also, $\left(\sigma_{N}(\mu)\right)^{\Upsilon}(m) \rightarrow \widehat{\mu}(m)$ as $N \rightarrow \infty$ for $m \in \mathbb{Z}$, so $\sigma_{N}(\mu) \rightarrow \mu$ wk $^{*}$ in $\Xi_{\mathcal{B}}^{*}$ as $N \rightarrow \infty$ by Theorem 2.1 .

When $\left(L^{1}\right)^{*}$ and $L^{\infty}$ are identified via the duality

$$
\langle f, g\rangle=\frac{1}{2 \pi} \int_{\mathbb{T}} f(t) g(-t) d t \quad\left(f \in L^{1}, g \in L^{\infty}\right),
$$

the functional $\xi_{n}$ is identified with the function $\alpha^{n}$. Hence

$$
\Xi_{L^{1}}={\overline{\operatorname{span}\left\{\alpha^{n}: n \in \mathbb{Z}\right\}}}^{L^{\infty}}=\mathcal{C}
$$

and thus

$$
\Xi_{L^{1}}^{*}=\mathcal{M} \text {. }
$$

In the general case, we shall now see that $\Xi_{\mathcal{B}}^{*}$ can be regarded as a subspace of $\mathcal{M}$. We first prove a more general version of the result.

Proposition 2.4. Let $\mathcal{B}_{1}$ and $\mathcal{B}_{2}$ be homogeneous Banach spaces on $\mathbb{T}$ and suppose that $\mathcal{B}_{1}$ is continuously embedded in $\mathcal{B}_{2}$. Let $\iota: \mathcal{B}_{1} \rightarrow \mathcal{B}_{2}$ be the inclusion map. Then $\rho=\left.\iota^{*}\right|_{\mathcal{B}_{2}}$ maps $\Xi_{\mathcal{B}_{2}}$ into a dense subset of $\Xi_{\mathcal{B}_{1}}$ and

$$
\rho^{*}: \Xi_{\mathcal{B}_{1}}^{*} \rightarrow \Xi_{\mathcal{B}_{2}}^{*}
$$

is 1-1. Moreover, if $\mathcal{B}_{1}$ is dense in $\mathcal{B}_{2}$, then $\rho$ is 1 -1 and the range of $\rho^{*}$ is $\mathrm{wk}^{*}$ dense in $\Xi_{\mathcal{B}_{2}}^{*}$.

Proof: We have $\rho\left(\xi_{n}\right)=\xi_{n}$ for $n \in \mathbb{Z}$, so $\rho$ maps $\Xi_{\mathcal{B}_{2}}$ into $\Xi_{\mathcal{B}_{1}}$ and has dense range. Hence $\rho^{*}$ is $1-1$. If $\mathcal{B}_{1}$ is dense in $\mathcal{B}_{2}$, then $\rho$ is clearly $1-1$ and the range of $\rho^{*}$ is thus $\mathrm{wk}^{*}$ dense in $\Xi_{\mathcal{B}_{2}}^{*}$.

With $\mathcal{B}_{2}=L^{1}$, we obtain the following.

Corollary 2.5. Let $\mathcal{B}$ be a homogeneous Banach space on $\mathbb{T}$ and let $\iota: \mathcal{B} \rightarrow L^{1}$ be the inclusion map. Then $\rho=\left.\iota^{*}\right|_{\mathcal{C}}$ maps $\mathcal{C}$ into a dense subset of $\Xi_{\mathcal{B}}$ and

$$
\rho^{*}: \Xi_{\mathcal{B}}^{*} \rightarrow \mathcal{M}
$$

is 1-1. Moreover, if $\mathcal{B}$ is dense in $L^{1}$, then $\rho$ is $1-1$ and the range of $\rho^{*}$ is $\mathrm{wk}^{*}$ dense in $\mathcal{M}$. 
From now on, we consider $\Xi_{\mathcal{B}}^{*}$ as a subspace of $\mathcal{M}$. For $\mu \in \Xi_{\mathcal{B}}^{*}$ and $s \in \mathbb{T}$, the translate $\mu_{s}$ is defined as a measure by $\mu_{s}(E)=\mu(E-s)$ for measurable sets $E \subseteq \mathbb{T}$. Since $\sigma_{N}\left(\mu_{s}\right)=\sigma_{N}(\mu)_{s}$ is a bounded sequence in $\mathcal{B}$ and since $\widehat{\sigma_{N}\left(\mu_{s}\right)}(m) \rightarrow \widehat{\mu_{s}}(m)$ as $N \rightarrow \infty$ for $m \in \mathbb{Z}$, we deduce that $\sigma_{N}\left(\mu_{s}\right) \rightarrow \mu_{s} \mathrm{wk}^{*}$ in $\Xi_{\mathcal{B}}^{*}$ as $N \rightarrow \infty$, so $\mu_{s} \in \Xi_{\mathcal{B}}^{*}$ with $\left\|\mu_{s}\right\|_{\Xi_{\mathcal{B}}^{*}}=\|\mu\|_{\Xi_{\mathcal{B}}^{*}}$. (Alternatively, $\mu_{s} \in \Xi_{\mathcal{B}}^{*}$ could be defined by $\left\langle\varphi, \mu_{s}\right\rangle=\left\langle\varphi_{s}, \mu\right\rangle\left(\varphi \in \Xi_{\mathcal{B}}\right)$.)

As a special case of strong Bochner integrals ([10, Chapter 3]), recall the following definition. Let $\mathcal{Z}$ be a Banach space and let $h: \mathbb{T} \rightarrow \mathcal{Z}^{*}$ be a $\mathrm{wk}^{*}$ measurable map (that is, the map $t \mapsto\langle z, h(t)\rangle(\mathbb{T} \rightarrow \mathbb{C}$ ) is measurable for every $z \in \mathcal{Z}$ ) with $\int_{\mathbb{T}}\|h(t)\| d t<\infty$. Define the wk ${ }^{*}$ Bochner integral $\int_{\mathbb{T}} h(t) d t \in \mathcal{Z}^{*}$ by

$$
\left\langle z, \int_{\mathbb{T}} h(t) d t\right\rangle=\int_{\mathbb{T}}\langle z, h(t)\rangle d t
$$

for $z \in \mathcal{Z}$. Also, for $f \in L^{1}$ and $\mu \in \mathcal{M}$, let

$$
(f * \mu)(t)=\frac{1}{2 \pi} \int_{\mathbb{T}} f(t-s) d \mu(s) \quad(t \in \mathbb{T})
$$

be the convolution of $f$ and $\mu$.

Theorem 2.6. Let $\mathcal{B}$ be a homogeneous Banach space on $\mathbb{T}$ and let $\mu \in$ $\Xi_{\mathcal{B}}^{*}$. Then the map $s \mapsto \mu_{s}\left(\mathbb{T} \rightarrow \Xi_{\mathcal{B}}^{*}\right)$ is $\mathrm{wk}^{*}$ continuous, and, for $f \in L^{1}$, the integral

$$
\frac{1}{2 \pi} \int_{\mathbb{T}} f(s) \mu_{s} d s
$$

exists as a $\mathrm{wk}^{*}$ Bochner integral in $\Xi_{\mathcal{B}}^{*}$ and equals $f * \mu$. Also, $\mu_{s} \rightarrow \mu$ in $\Xi_{\mathcal{B}}^{*}$ as $s \rightarrow 0$ if and only if $\mu \in \mathcal{B}$.

Proof: We have $\left\|\mu_{s}\right\|_{\Xi_{\mathcal{B}}^{*}}=\|\mu\|_{\Xi_{\mathcal{B}}^{*}}$ for $s \in \mathbb{T}$ and $\widehat{\mu_{s}}(n)=e^{-i n s} \widehat{\mu}(n) \rightarrow$ $\widehat{\mu}(n)$ as $s \rightarrow 0$ for $n \in \mathbb{Z}$, so $\mu_{s} \rightarrow \mu \mathrm{wk}^{*}$ in $\Xi_{\mathcal{B}}^{*}$ as $s \rightarrow 0$ by Theorem 2.1. For $f \in L^{1}$, it thus follows that $\int_{\mathbb{T}} f(s) \mu_{s} d s$ exists as a wk* Bochner integral in $\Xi_{\mathcal{B}}^{*}$. Furthermore, Bochner integrals commute with continuous linear functionals, so

$$
\begin{aligned}
\left(\frac{1}{2 \pi} \int_{\mathbb{T}} f(s) \mu_{s} d s\right) \uparrow(n) & =\frac{1}{2 \pi} \int_{\mathbb{T}}\left\langle\xi_{n}, f(s) \mu_{s}\right\rangle d s=\frac{1}{2 \pi} \int_{\mathbb{T}} f(s) \widehat{\mu_{s}}(n) d s \\
& =\widehat{\mu}(n) \frac{1}{2 \pi} \int_{\mathbb{T}} f(s) e^{-i n s} d s=\widehat{f}(n) \widehat{\mu}(n)
\end{aligned}
$$

for $n \in \mathbb{Z}$. Consequently $1 /(2 \pi) \int_{\mathbb{T}} f(s) \mu_{s} d s=f * \mu$.

Let $\mathcal{Z}$ be the space of those $\mu \in \Xi_{\mathcal{B}}^{*}$ for which $\mu_{s} \rightarrow \mu$ in $\Xi_{\mathcal{B}}^{*}$ as $s \rightarrow 0$. Then $\mathcal{B} \subseteq \mathcal{Z}$. On the other hand, for $\mu \in \mathcal{Z}$, we have $\mu_{s} \rightarrow \mu$ in $\mathcal{M}$ as $s \rightarrow 0$, so it follows from [9, V.19.27] that $\mu \in L^{1}$. Also, $\mathcal{Z}$ is 
closed in $\Xi_{\mathcal{B}}^{*}$, so we deduce that $\mathcal{Z}$ is a homogeneous Banach space on $\mathbb{T}$. Hence the trigonometric polynomials in $\mathcal{Z}$ are dense in $\mathcal{Z}$, so $\mathcal{Z} \subseteq \mathcal{B}$ by Lemma 2.2 .

Our aim now is to describe the spaces $\Xi_{\mathcal{B}}^{*}$ as so-called prehomogeneous Banach spaces on $\mathbb{T}$. To suit our purpose, we have weakened condition (i) in the following definition compared to the definition in [12, p. 64] (where it is required that $\mathcal{Y}$ contains every trigonometric polynomial).

A Banach space $\mathcal{Y}$ continuously embedded in $\mathcal{M}$ is called a prehomogeneous Banach space on $\mathbb{T}$ if the following conditions are satisfied.

(i) If $\mu \in \mathcal{Y}$ and $\widehat{\mu}(n) \neq 0$ for some $n \in \mathbb{Z}$, then $\alpha^{n} \in \mathcal{Y}$. Moreover, for every trigonometric polynomial $p$ in $\mathcal{Y}$, we have $\left\|p_{s}\right\|_{\mathcal{Y}}=\|p\|_{\mathcal{Y}}$ for $s \in \mathbb{T}$ and $p_{s} \rightarrow p$ in $\mathcal{Y}$ as $s \rightarrow 0$.

(ii) For every trigonometric polynomial $p$ and $\mu \in \mathcal{Y}$, we have $\| p *$ $\mu\left\|_{\mathcal{Y}} \leq\right\| p\left\|_{L^{1}}\right\| \mu \|_{\mathcal{Y}}$. (Since $p * \mu=\sum \widehat{p}(n) \widehat{\mu}(n) \alpha^{n}$, we have $p * \mu \in \mathcal{Y}$ by (i).)

(iii) There is a topology, $\tau$, on $\mathcal{Y}$ such that the unit ball of $\mathcal{Y}$ is $\tau$ compact and such that the map $\xi_{n}: \mathcal{Y} \rightarrow \mathbb{C}$ is $\tau$ continuous for $n \in \mathbb{Z}$.

The following is [12, Exercises 2 and 3, p. 64].

Lemma 2.7. Let $\mathcal{Y}$ be a prehomogeneous Banach space on $\mathbb{T}$.

(a) A measure $\mu \in \mathcal{M}$ belongs to $\mathcal{Y}$ if and only if $\left(\sigma_{N}(\mu)\right)$ is a bounded sequence in $\mathcal{Y}$.

(b) The closure $\mathcal{Y}_{h}$ in $\mathcal{Y}$ of the trigonometric polynomials in $\mathcal{Y}$ is a homogeneous Banach space on $\mathbb{T}$.

Proof: (a) Let $\mu \in \mathcal{Y}$. Since $\sigma_{N}(\mu)=K_{N} * \mu$, where $\left(K_{N}\right)$ is the Fejér kernel $\left(\left[13\right.\right.$, p. 12]), and since $\left\|K_{N}\right\|_{L^{1}}=1$ for $N \in \mathbb{N}$, it follows from (ii) that $\left(\sigma_{N}(\mu)\right)$ is a bounded sequence in $\mathcal{Y}$. Conversely, if $\mu \in \mathcal{M}$ and $\left(\sigma_{N}(\mu)\right)$ is a bounded sequence in $\mathcal{Y}$, then it has a $\tau$ clusterpoint $\nu \in \mathcal{Y}$ as $N \rightarrow \infty$. Since $\xi_{n}$ is $\tau$ continuous for $n \in \mathbb{Z}$, we deduce that $\mu=\nu \in \mathcal{Y}$.

(b) We have $\mathcal{Y}_{h} \subseteq L^{1}$ since $L^{1}$ is closed in $\mathcal{M}$. For $f \in \mathcal{Y}_{h}$, it follows from (i) that $\left\|f_{s}\right\|_{\mathcal{Y}}=\|f\|_{\mathcal{Y}}(s \in \mathbb{T})$ and that $f_{s} \rightarrow f$ in $\mathcal{Y}$ as $s \rightarrow 0$. Hence $\mathcal{Y}_{h}$ is a homogeneous Banach space on $\mathbb{T}$.

We shall now see that $\Xi_{\mathcal{B}}^{*}$ is a prehomogeneous Banach space on $\mathbb{T}$.

Theorem 2.8. Let $\mathcal{B}$ be a homogeneous Banach space on $\mathbb{T}$. Equipped with the $\mathrm{wk}^{*}$ topology, $\Xi_{\mathcal{B}}^{*}$ is a prehomogeneous Banach space on $\mathbb{T}$ and

$$
\left(\Xi_{\mathcal{B}}^{*}\right)_{h}=\mathcal{B}
$$


Proof: Condition (i) follows from Lemma 2.2. Let $p$ be a trigonometric polynomial and let $\mu \in \Xi_{\mathcal{B}}^{*}$. Then $p * \mu=1 /(2 \pi) \int_{\mathbb{T}} p(s) \mu_{s} d s$ exists as a $\mathrm{wk}^{*}$ Bochner integral in $\Xi_{\mathcal{B}}^{*}$ by Theorem 2.6. Hence $\|p * \mu\|_{\Xi_{\mathcal{B}}^{*}} \leq$ $\|p\|_{L^{1}}\|\mu\|_{\Xi_{\mathcal{B}}^{*}}$, so (ii) holds. Moreover, (iii) follows from Alaoglu's theorem and Theorem 2.1. Since $\mathcal{B}$ is the closure of the trigonometric polynomials in $\mathcal{B}$ and is closed in $\Xi_{\mathcal{B}}^{*}$, we deduce from Lemma 2.2 that $\left(\Xi_{\mathcal{B}}^{*}\right)_{h}=\mathcal{B}$.

For $\mu \in \Xi_{\mathcal{B}}^{*}$, we have $\sigma_{N}(\mu) \in \mathcal{B}$ by Lemma 2.2. The next corollary is an immediate consequence of the two previous results and gives a useful characterization of $\Xi_{\mathcal{B}}^{*}$.

Corollary 2.9. Let $\mathcal{B}$ be a homogeneous Banach space on $\mathbb{T}$ and let $\mu \in \mathcal{M}$. Then $\mu \in \Xi_{\mathcal{B}}^{*}$ if and only if $\left(\sigma_{N}(\mu)\right)$ is a bounded sequence in $\mathcal{B}$.

Finally, we can now prove that the correspondence $\mathcal{B} \mapsto \Xi_{\mathcal{B}}^{*}$ is bijective.

Theorem 2.10. The maps

$$
\mathcal{B} \mapsto \Xi_{\mathcal{B}}^{*}
$$

$(\mathcal{B}$ a homogeneous Banach space on $\mathbb{T}$ ) and

$$
\mathcal{Y} \mapsto \mathcal{Y}_{h}
$$

$(\mathcal{Y}$ a prehomogeneous Banach space on $\mathbb{T}$ ) define a bijective correspondence between the class of homogeneous Banach spaces on $\mathbb{T}$ and the class of prehomogeneous Banach spaces on $\mathbb{T}$.

Proof: By Theorem 2.8, we have $\left(\Xi_{\mathcal{B}}^{*}\right)_{h}=\mathcal{B}$, when $\mathcal{B}$ is a homogeneous Banach space on $\mathbb{T}$. Now, let $\mathcal{Y}$ be a prehomogeneous Banach space on $\mathbb{T}$. For $\mu \in \mathcal{M}$, the previous corollary implies that $\mu \in \Xi_{\mathcal{Y}_{h}}^{*}$ if and only if $\left(\sigma_{N}(\mu)\right)$ is a bounded sequence in $\mathcal{Y}_{h}$. However, this condition is also equivalent to $\mu \in \mathcal{Y}$ by Lemma 2.7. Hence $\Xi_{\mathcal{Y}_{h}}^{*}=\mathcal{Y}$, which proves the result.

\section{Homogeneous Banach algebras}

The simplest example of a homogeneous Banach space on $\mathbb{T}$ which is also a Banach algebra under pointwise multiplication is the algebra $\mathcal{C}$. We have $\mathcal{C}^{*}=\mathcal{M}, \Xi_{\mathcal{C}}=L^{1}$ and thus

$$
\Xi_{\mathcal{C}}^{*}=L^{\infty},
$$

so $\Xi_{\mathcal{C}}^{*}$ is closed under pointwise multiplication. This is true in general. 
Theorem 3.1. Let $\mathcal{B}$ be a homogeneous Banach space on $\mathbb{T}$ and suppose that $\mathcal{B}$ is a Banach algebra under pointwise multiplication. Then

(a) $\mathcal{B} \subseteq \mathcal{C}$.

(b) $\Xi_{\mathcal{B}}^{*} \subseteq L^{\infty}$ and $\Xi_{\mathcal{B}}^{*}$ is a Banach algebra under pointwise multiplication.

(c) If a homogeneous Banach space $\mathcal{L}$ on $\mathbb{T}$ is a Banach $\mathcal{B}$-module under pointwise multiplication, then $\Xi_{\mathcal{L}}^{*}$ is a Banach $\Xi_{\mathcal{B}}^{*}$-module under pointwise multiplication.

Proof: Let $f \in \mathcal{B}$ with $\|f\|_{\mathcal{B}} \leq 1$. Then $\left(f^{n}\right)$ is bounded in $L^{1}$, so $f \in L^{\infty}$ with $\|f\|_{\infty} \leq 1$. Hence $\mathcal{B} \subseteq L^{\infty}$ and the inclusion is continuous. Moreover, $\sigma_{N}(f) \rightarrow f$ in $\mathcal{B}$ and thus in $L^{\infty}$ as $N \rightarrow \infty$, so we deduce that $f \in \mathcal{C}$. Consequently $\mathcal{B} \subseteq \mathcal{C}$ and thus $\Xi_{\mathcal{B}}^{*} \subseteq L^{\infty}$ by Proposition 2.4. Now, let $f \in \Xi_{\mathcal{B}}^{*}$ and $g \in \Xi_{\mathcal{L}}^{*}$. Then

$$
\left\|\sigma_{N}(f) \sigma_{N}(g)\right\|_{\mathcal{L}} \leq\left\|\sigma_{N}(f)\right\|_{\mathcal{B}} \cdot\left\|\sigma_{N}(g)\right\|_{\mathcal{L}} \leq\|f\|_{\Xi_{\mathcal{B}}^{*}} \cdot\|g\|_{\Xi_{\mathcal{L}}^{*}}
$$

for $N \in \mathbb{N}$, so the sequence $\left(\sigma_{N}(f) \sigma_{N}(g)\right)$ has a $\mathrm{wk}^{*}$ cluster point $h \in \Xi_{\mathcal{L}}^{*}$ as $N \rightarrow \infty$. Since $\left(\sigma_{N}(f) \sigma_{N}(g)\right)^{\uparrow}(m) \rightarrow \widehat{f g}(m)$ as $N \rightarrow \infty$ for $m \in \mathbb{Z}$, we deduce that $f g=h \in \Xi_{\mathcal{L}}^{*}$, with

$$
\|f g\|_{\Xi_{\mathcal{L}}^{*}} \leq\|f\|_{\Xi_{\mathcal{B}}^{*}} \cdot\|g\|_{\Xi_{\mathcal{L}}^{*}}
$$

Considering $\mathcal{B}$ as a Banach $\mathcal{B}$-module, this shows that $\Xi_{\mathcal{B}}^{*}$ is a Banach algebra under pointwise multiplication, and the result follows.

It is well known that the dual space $\mathcal{B}^{*}$ of a commutative Banach algebra $\mathcal{B}$ is a commutative Banach $\mathcal{B}$-module under the action $\langle a, b \varphi\rangle=$ $\langle a b, \varphi\rangle\left(a, b \in \mathcal{B}, \varphi \in \mathcal{B}^{*}\right)$. The fact that $L^{1}$ is a Banach $L^{\infty}$-module thus states that $\Xi_{\mathcal{C}}$ is a $\Xi_{\mathcal{C}}^{*}$-submodule of $\Xi_{\mathcal{C}}^{* *}$, and we shall see that this holds in general.

Proposition 3.2. Let $\mathcal{B}$ be a homogeneous Banach space on $\mathbb{T}$ which is also a Banach algebra under pointwise multiplication. Then $\Xi_{\mathcal{B}}$ is a closed $\Xi_{\mathcal{B}}^{*}$-submodule of $\Xi_{\mathcal{B}}^{* *}$.

Proof: Let $\rho: L^{1} \rightarrow \Xi_{\mathcal{B}}$ be as in Proposition 2.4 (with $\mathcal{B}_{2}=\mathcal{C}$ ). For $f \in L^{1}$ and $g \in \Xi_{\mathcal{B}}^{*}$, it is easily verified that $g \rho(f)=\rho^{* *}\left(\rho^{*}(g) f\right)$ in $\Xi_{\mathcal{B}}^{* *}$. Since $\rho^{*}(g) f \in L^{1}$, we thus have $g \rho(f) \in \Xi_{\mathcal{B}}$, and the result follows since $\rho$ has dense range in $\Xi_{\mathcal{B}}$.

As a standard corollary, we obtain the following.

Corollary 3.3. Let $\mathcal{B}$ be a homogeneous Banach space on $\mathbb{T}$ which is also a Banach algebra under pointwise multiplication. Then multiplication is separately $\mathrm{wk}^{*}$ continuous in $\Xi_{\mathcal{B}}^{*}$. 
Proof: Let $\left(f_{i}\right)$ be a net in $\Xi_{\mathcal{B}}^{*}$ that converges wk ${ }^{*}$ to 0 , and let $g \in \Xi_{\mathcal{B}}^{*}$ and $\varphi \in \Xi_{\mathcal{B}}$. Then $g \varphi \in \Xi_{\mathcal{B}}$ by the previous proposition, so we deduce that

$$
\left\langle\varphi, g f_{i}\right\rangle=\left\langle g f_{i}, \varphi\right\rangle=\left\langle f_{i}, g \varphi\right\rangle=\left\langle g \varphi, f_{i}\right\rangle \rightarrow 0
$$

as required.

Remark. Multiplication need not be $\mathrm{wk}^{*}$ continuous in $\Xi_{\mathcal{B}}^{*}$. In $L^{\infty}$, we have $\alpha^{n} \rightarrow 0$ wk $^{*}$ as $|n| \rightarrow \infty$, but $\alpha^{n} \alpha^{-n}=1$ for $n \in \mathbb{N}$.

We say that a homogeneous Banach space $\mathcal{B}$ on $\mathbb{T}$ is a homogeneous Banach algebra on $\mathbb{T}$ if $\mathcal{B}$ is a Banach algebra under pointwise multiplication and the character space of $\mathcal{B}$ is $\mathbb{T}$. We shall use the inclusion $\Xi_{\mathcal{B}}^{*} \subseteq L^{\infty}$ to show that $\mathcal{C}$ is the only homogeneous Banach algebra on $\mathbb{T}$ in which every closed ideal has an bounded approximate identity with a common bound. The following result as well as its proof is similar to $[\mathbf{4}$, Lemma XVII.2.1].

Proposition 3.4. Let $\mathcal{Y}$ be a Banach algebra continuously embedded in $L^{\infty}$ and suppose that there exists a constant $C$ such that $1_{V} \in \mathcal{Y}$ with $\left\|1_{V}\right\|_{\mathcal{Y}} \leq C$ for every open set $V \subseteq \mathbb{T}$. Then $\mathcal{C} \subseteq \mathcal{Y}$ and $\|\cdot\|_{\mathcal{Y}}$ is equivalent to the uniform norm on $\mathcal{C}$.

Proof: Let $V_{1}, \ldots, V_{N}$ be pairwise disjoint open sets in $\mathbb{T}$, let $a_{1}, \ldots, a_{N}$ be complex numbers and consider the function

$$
f=\sum_{n=1}^{N} a_{n} 1_{V_{n}} .
$$

Then $\|f\|_{\infty}=\sup _{1 \leq n \leq N}\left|a_{n}\right|$. For $\varphi \in \mathcal{Y}^{*}$ with $\|\varphi\|_{\mathcal{Y}^{*}} \leq 1$, we have

$$
|\langle f, \varphi\rangle| \leq \sum_{n=1}^{N}\left|a_{n}\right| \cdot\left|\left\langle 1_{V_{n}}, \varphi\right\rangle\right| \leq\|f\|_{\infty} \sum_{n=1}^{N}\left|\left\langle 1_{V_{n}}, \varphi\right\rangle\right| .
$$

Denoting the real and imaginary part of $\varphi$ by $\varphi_{1}$ and $\varphi_{2}$, we have

$$
\begin{aligned}
\sum_{n=1}^{N}\left|\left\langle 1_{V_{n}}, \varphi\right\rangle\right| \leq & \sum_{\left\langle 1_{V_{n}}, \varphi_{1}\right\rangle \geq 0}\left\langle 1_{V_{n}}, \varphi_{1}\right\rangle-\sum_{\left\langle 1_{V_{n}}, \varphi_{1}\right\rangle<0}\left\langle 1_{V_{n}}, \varphi_{1}\right\rangle \\
& +\sum_{\left\langle 1_{V_{n}}, \varphi_{2}\right\rangle \geq 0}\left\langle 1_{V_{n}}, \varphi_{2}\right\rangle-\sum_{\left\langle 1_{V_{n}}, \varphi_{2}\right\rangle<0}\left\langle 1_{V_{n}}, \varphi_{2}\right\rangle \\
= & \left\langle 1_{W_{1+}}, \varphi_{1}\right\rangle-\left\langle 1_{W_{1-}}, \varphi_{1}\right\rangle+\left\langle 1_{W_{2+}}, \varphi_{2}\right\rangle-\left\langle 1_{W_{2-}}, \varphi_{2}\right\rangle,
\end{aligned}
$$

where $W_{1+}, W_{1-}, W_{2+}$ and $W_{2-}$ are open sets. Hence

$$
|\langle f, \varphi\rangle| \leq 4 C\|f\|_{\infty},
$$


so we deduce that

$$
\|f\|_{\mathcal{Y}} \leq 4 C\|f\|_{\infty} .
$$

Consequently the two norms are equivalent on

$$
\operatorname{span}\left\{1_{V}: V \subseteq \mathbb{T} \text { is open }\right\} .
$$

Let $f \in \mathcal{C}$ with $0 \leq f \leq 1$. For $N \in \mathbb{N}$, let

$$
U_{N n}=\{t \in \mathbb{T}: f(t)>n / N\} \quad(n=0, \ldots, N-1),
$$

and let

$$
f_{N}=\frac{1}{N} \sum_{n=0}^{N-1} 1_{U_{N n}}
$$

Then $\left\|f-f_{N}\right\|_{\infty} \leq 1 / N$, so we deduce that

$$
\mathcal{C} \subseteq \overline{\operatorname{span}}\left\{1_{V}: V \subseteq \mathbb{T} \text { is open }\right\},
$$

and the result follows.

Theorem 3.5. The algebra $\mathcal{C}$ is the only homogeneous Banach algebra on $\mathbb{T}$ in which there exists a constant $C$ such that every closed ideal has a approximate identity bounded by $C$.

Proof: Let $\mathcal{B}$ be homogeneous Banach algebra on $\mathbb{T}$ and suppose that there exists a constant $C$ such that every closed ideal in $\mathcal{B}$ has a bounded approximate identity bounded by $C$. Let $E \subseteq \mathbb{T}$ be a closed set and let $\left(f_{n}\right)$ be an approximate identity bounded by $C$ for the closed ideal $\{f \in \mathcal{B}: f=0$ on $E\}$. Then $f_{n} \rightarrow 1$ uniformly on compact sets in $\mathbb{T} \backslash E$ and $\left(f_{n}\right)$ is uniformly bounded, so

$$
\widehat{f_{n}}(m) \rightarrow \widehat{1_{\mathbb{T} \backslash E}}(m) \quad(m \in \mathbb{Z})
$$

as $n \rightarrow \infty$. On the other hand, let $f$ be a wk ${ }^{*}$ cluster point in $\Xi_{\mathcal{B}}^{*}$ of the sequence $\left(f_{n}\right)$ and let $\left(f_{n_{i}}\right)$ be a subnet of $\left(f_{n}\right)$ which converges wk ${ }^{*}$ to $f$. Then

$$
\widehat{f_{n_{i}}}(m) \rightarrow \widehat{f}(m) \quad(m \in \mathbb{Z}),
$$

so we deduce that $1_{\mathbb{T} \backslash E}=f \in \Xi_{\mathcal{B}}^{*}$ with $\left\|1_{\mathbb{T} \backslash E}\right\|_{\Xi_{\mathcal{B}}^{*}} \leq C$. Theorem 3.1 and the previous proposition thus imply that the norm $\|\cdot\|_{\mathcal{B}}$ is equivalent to the uniform norm on $\mathcal{B}$, so $\mathcal{B}$ is a closed subalgebra of $\mathcal{C}$. Since $\mathcal{B}$ is a homogeneous Banach algebra on $\mathbb{T}$, it thus follows that $\mathcal{B}=\mathcal{C}$.

We do not know whether there exists a homogeneous Banach algebra on $\mathbb{T}$ other than $\mathcal{C}$ in which every closed ideal has a bounded approximate identity. 


\section{Multipliers}

Let $X_{1}$ and $X_{2}$ be Banach spaces continuously embedded in $\mathcal{M}$. We say that a linear operator $T: X_{1} \rightarrow X_{2}$ is a multiplier if there exists a sequence $(\widehat{T}(n))$ such that

$$
\widehat{T \mu}=\widehat{T} \widehat{\mu} \quad\left(\mu \in X_{1}\right) .
$$

Such an operator is automatically continuous. We denote the space of multipliers from $X_{1}$ to $X_{2}$ by $\left(X_{1}, X_{2}\right)$. (See [5] or [14] for general information on multipliers and for descriptions of $\left(X_{1}, X_{2}\right)$ for various choices of $X_{1}$ and $X_{2}$.) For homogeneous Banach spaces, we have the following well-known result. Observe that every homogeneous Banach space $\mathcal{B}$ on $\mathbb{T}$ is a Banach $\mathcal{M}$-module for the convolution product since $\mu * f=(1 /(2 \pi)) \int_{\mathbb{T}} f_{s} d \mu(s) \in \mathcal{B}$ for $\mu \in \mathcal{M}$ and $f \in \mathcal{B}$.

Proposition 4.1. For homogeneous Banach spaces $\mathcal{B}_{1}$ and $\mathcal{B}_{2}$ on $\mathbb{T}$ and a linear operator $T: \mathcal{B}_{1} \rightarrow \mathcal{B}_{2}$, the following conditions are equivalent.

(a) $T \in\left(\mathcal{B}_{1}, \mathcal{B}_{2}\right)$.

(b) $T(\mu * f)=\mu * T f \quad\left(\mu \in \mathcal{M}, f \in \mathcal{B}_{1}\right)$.

(c) $T R_{s}=R_{s} T \quad(s \in \mathbb{T})$.

For the homogeneous Banach spaces $\mathcal{B}$ considered in this section, we assume, for simplicity, that, for every $n \in \mathbb{Z}$, there exists $f \in \mathcal{B}$ such that $\widehat{f}(n) \neq 0$.

Proposition 4.2. Let $\mathcal{B}_{1}$ and $\mathcal{B}_{2}$ be homogeneous Banach spaces on $\mathbb{T}$. Every $T \in\left(\mathcal{B}_{1}, \mathcal{B}_{2}\right)$ extends uniquely by wk $^{*}$ continuity to $\widetilde{T} \in$ $\left(\Xi_{\mathcal{B}_{1}}^{*}, \Xi_{\mathcal{B}_{2}}^{*}\right)$. Conversely, every $S \in\left(\Xi_{\mathcal{B}_{1}}^{*}, \Xi_{\mathcal{B}_{2}}^{*}\right)$ maps $\mathcal{B}_{1}$ into $\mathcal{B}_{2}$, so $\left.S\right|_{\mathcal{B}_{1}} \in\left(\mathcal{B}_{1}, \mathcal{B}_{2}\right)$. Moreover, $\left(\mathcal{B}_{1}, \mathcal{B}_{2}\right)=\left(\mathcal{B}_{1}, \Xi_{\mathcal{B}_{2}}^{*}\right)$.

Proof: For $T \in\left(\mathcal{B}_{1}, \mathcal{B}_{2}\right)$ and $n \in \mathbb{Z}$, we have

$$
\left\langle f, T^{*} \xi_{n}\right\rangle=\widehat{T}(n) \widehat{f}(n)=\left\langle f, \widehat{T}(n) \xi_{n}\right\rangle \quad\left(f \in \mathcal{B}_{1}\right),
$$

so $T^{*} \xi_{n}=\widehat{T}(n) \xi_{n}$. Hence $T^{*}\left(\Xi_{\mathcal{B}_{2}}\right) \subseteq \Xi_{\mathcal{B}_{1}}$ and $\widetilde{T}=\left(\left.T^{*}\right|_{\Xi_{\mathcal{B}_{2}}}\right)^{*}$ is the required extension. For $S \in\left(\Xi_{\mathcal{B}_{1}}^{*}, \Xi_{\mathcal{B}_{2}}^{*}\right)$, we have $S \alpha^{n}=\widehat{S}(n) \alpha^{n}(n \in \mathbb{Z})$, so $S\left(\mathcal{B}_{1}\right) \subseteq \mathcal{B}_{2}$. The same argument proves the last statement.

Remark. For linear operators $S: \Xi_{\mathcal{B}_{1}}^{*} \rightarrow \Xi_{\mathcal{B}_{2}}^{*}$, we still have (a) $\Leftrightarrow(\mathrm{b}) \Rightarrow(\mathrm{c})$ in Proposition 4.1, but not necessarily (c) $\Rightarrow(\mathrm{b})$; see, for example, [19]. 
Figà-Talamanca and Gaudry $([\mathbf{6}],[\mathbf{7}])$ have shown that $\left(L^{p}, L^{q}\right)$ $(1 \leq p, q<\infty)$ is a dual space. We shall use a result of Rieffel $([\mathbf{1 8}])$ to show that this holds for homogeneous Banach spaces in general. For Banach spaces $X$ and $Y$, denote their projective tensor product by $X \otimes_{\gamma} Y$. When $X$ and $Y$ are Banach $L^{1}$-modules, let

$$
K=\overline{\operatorname{span}}\left\{f x \otimes y-x \otimes f y: f \in L^{1}, x \in X, y \in Y\right\}
$$

and let

$$
X \otimes_{L^{1}} Y=\left(X \otimes_{\gamma} Y\right) / K .
$$

Then every $\rho \in X \otimes_{L^{1}} Y$ has an expansion

$$
\rho=\sum_{n=1}^{\infty} x_{n} \otimes y_{n}
$$

with $\sum_{n=1}^{\infty}\left\|x_{n}\right\|_{X} \cdot\left\|y_{n}\right\|_{Y}<\infty$, and

$$
\|\rho\|_{X \otimes_{L^{1}} Y}=\inf \left\{\sum_{n=1}^{\infty}\left\|x_{n}\right\|_{X} \cdot\left\|y_{n}\right\|_{Y}: \rho=\sum_{n=1}^{\infty} x_{n} \otimes y_{n}\right\}
$$

is the norm on $X \otimes_{L^{1}} Y$.

Theorem 4.3. Let $\mathcal{B}_{1}$ and $\mathcal{B}_{2}$ be homogeneous Banach spaces on $\mathbb{T}$ and define

$$
\Phi:\left(\mathcal{B}_{1}, \mathcal{B}_{2}\right) \rightarrow\left(\mathcal{B}_{1} \otimes_{L^{1}} \Xi_{\mathcal{B}_{2}}\right)^{*}
$$

by

$$
\langle f \otimes \varphi, \Phi(T)\rangle=\langle T f, \varphi\rangle \quad\left(f \in \mathcal{B}_{1}, \varphi \in \Xi_{\mathcal{B}_{2}}, T \in\left(\mathcal{B}_{1}, \mathcal{B}_{2}\right)\right) .
$$

Then $\Phi$ is an isometric isomorphism, and $\Phi$ is a homeomorphism between the closed unit balls equipped with the weak operator and the $\mathrm{wk}^{*}$ topology respectively.

Proof: It follows from [18] that the map $\widetilde{\Phi}:\left(\mathcal{B}_{1}, \Xi_{\mathcal{B}_{2}}^{*}\right) \rightarrow\left(\mathcal{B}_{1} \otimes_{L^{1}} \Xi_{\mathcal{B}_{2}}\right)^{*}$ defined by $\langle f \otimes \varphi, \widetilde{\Phi}(T)\rangle=\langle\varphi, T f\rangle$ for $f \in \mathcal{B}_{1}, \varphi \in \Xi_{\mathcal{B}_{2}}$ and $T \in\left(\mathcal{B}_{1}, \Xi_{\mathcal{B}_{2}}^{*}\right)$ is an isometric isomorphism, so $\Phi$ is an isometric isomorphism by the previous proposition. Let $\left(T_{i}\right)$ be a net in the closed unit ball of $\left(\mathcal{B}_{1}, \mathcal{B}_{2}\right)$ which converges to $T$ in the weak operator topology. Then

$$
\left\langle f \otimes \varphi, \Phi\left(T_{i}\right)\right\rangle \rightarrow\langle f \otimes \varphi, \Phi(T)\rangle \quad\left(f \in \mathcal{B}_{1}, \varphi \in \Xi_{\mathcal{B}_{2}}\right),
$$

and it follows that $\Phi\left(T_{i}\right) \rightarrow \Phi(T) \mathrm{wk}^{*}$. From [2, Proposition IX.5.5] (the proof given there carries over to Banach spaces), we deduce that the closed unit ball of $\left(\mathcal{B}_{1}, \mathcal{B}_{2}\right)$ is compact in the weak operator topology, so the result follows. 
We shall now show that the abstract space $\mathcal{B}_{1} \otimes_{L^{1}} \Xi_{\mathcal{B}_{2}}$ can be identified with a sequence space. For $f \in \mathcal{B}_{1}$ and $\varphi \in \Xi_{\mathcal{B}_{2}}$, define $f * \varphi$ as a sequence by $\widehat{f * \varphi}=\widehat{f} \widehat{\varphi}$, and let

$$
\mathcal{B}_{1} * \Xi_{\mathcal{B}_{2}}=\left\{\rho=\sum_{n=1}^{\infty} f_{n} * \varphi_{n}: \sum_{n=1}^{\infty}\left\|f_{n}\right\|_{\mathcal{B}_{1}} \cdot\left\|\varphi_{n}\right\|_{\Xi_{\mathcal{B}_{2}}}<\infty\right\}
$$

equipped with norm

$$
\|\rho\|_{\mathcal{B}_{1} * \Xi_{\mathcal{B}_{2}}}=\inf \left\{\sum_{n=1}^{\infty}\left\|f_{n}\right\|_{\mathcal{B}_{1}} \cdot\left\|\varphi_{n}\right\|_{\Xi_{\mathcal{B}_{2}}}: \rho=\sum_{n=1}^{\infty} f_{n} * \varphi_{n}\right\} .
$$

For $L^{p}$ spaces, the following was proved in [18, Theorem 3.3].

Proposition 4.4. There exists an isometric isomorphism $\Psi: \mathcal{B}_{1} \otimes_{L^{1}}$ $\Xi_{\mathcal{B}_{2}} \rightarrow \mathcal{B}_{1} * \Xi_{\mathcal{B}_{2}}$ such that $\Psi(f \otimes \varphi)=f * \varphi\left(f \in \mathcal{B}_{1}, \varphi \in \Xi_{\mathcal{B}_{2}}\right)$.

Proof: The map $\psi: \mathcal{B}_{1} \times \Xi_{\mathcal{B}_{2}} \rightarrow \mathcal{B}_{1} * \Xi_{\mathcal{B}_{2}}$ defined by $\psi(f, \varphi)=f * \varphi(f \in$ $\left.\mathcal{B}_{1}, \varphi \in \Xi_{\mathcal{B}_{2}}\right)$ is bilinear and continuous, so there is a continuous, linear map $\widetilde{\psi}: \mathcal{B}_{1} \otimes_{\gamma} \Xi_{\mathcal{B}_{2}} \rightarrow \mathcal{B}_{1} * \Xi_{\mathcal{B}_{2}}$ with $\widetilde{\psi}(f \otimes \varphi)=f * \varphi\left(f \in \mathcal{B}_{1}, \varphi \in \Xi_{\mathcal{B}_{2}}\right)$. Moreover, $\widetilde{\psi}$ is surjective and $K \subseteq \operatorname{ker} \widetilde{\psi}$. For $f \in \mathcal{B}_{1}$ and $\varphi \in \Xi_{\mathcal{B}_{2}}$, we have $f * \varphi=(1 /(2 \pi)) \int_{\mathbb{T}} f(s) \varphi_{s} d s \in \Xi_{\mathcal{B}_{2}}$, so it follows from the proof of $[\mathbf{1 8}$, Theorem 3.3] that ker $\widetilde{\psi} \subseteq K$, which finishes the proof.

Every trigonometric polynomial $p$ defines a multiplier $T_{p} \in\left(\mathcal{B}_{1}, \mathcal{B}_{2}\right)$ by $T_{p} f=p * f\left(f \in \mathcal{B}_{1}\right)$, which we identify with $p$. We have the following density results.

\section{Proposition 4.5.}

(a) For homogeneous Banach spaces $\mathcal{B}_{1}$ and $\mathcal{B}_{2}$ on $\mathbb{T}$, the unit ball of the trigonometric polynomials is strongly dense in the unit ball of $\left(\mathcal{B}_{1}, \mathcal{B}_{2}\right)$.

(b) For a homogeneous Banach space $\mathcal{B}$ on $\mathbb{T}$, the span of $\left\{R_{s}: s \in \mathbb{T}\right\}$ is strongly dense in $(\mathcal{B}, \mathcal{B})$.

Proof: (a) For $T \in\left(\mathcal{B}_{1}, \mathcal{B}_{2}\right)$, let

$$
\sigma_{N}(T)=\sum_{n=-N}^{N}\left(1-\frac{|n|}{N+1}\right) \widehat{T}(n) \alpha^{n} \quad(N \in \mathbb{N}) .
$$

Then $\sigma_{N}(T) f=\sigma_{N}(T f) \rightarrow T f$ in $\mathcal{B}_{2}$ as $N \rightarrow \infty$ for $f \in \mathcal{B}_{1}$ and $\left\|\sigma_{N}(T)\right\|_{\left(\mathcal{B}_{1}, \mathcal{B}_{2}\right)} \leq\|T\|_{\left(\mathcal{B}_{1}, \mathcal{B}_{2}\right)}(N \in \mathbb{N})$. 
(b) For $n \in \mathbb{Z}$ and $f \in \mathcal{B}$, the integral

$$
\alpha^{n} * f=\frac{1}{2 \pi} \int_{\mathbb{T}} R_{s} f \cdot e^{i n s} d s
$$

exists as a Bochner integral, so $\alpha^{n}$ belongs to the strong closure of $\operatorname{span}\left\{R_{s}: s \in \mathbb{T}\right\}$, and the conclusion thus follows from (a).

Finally, we shall see that $(\mathcal{B}, \mathcal{B})$ is the dual space of a homogeneous Banach space of continuous functions on $\mathbb{T}$. Let $\mathcal{W}$ be the Wiener algebra of absolutely convergent Fourier series on $\mathbb{T}$.

Corollary 4.6. Let $\mathcal{B}$ be a homogeneous Banach space on $\mathbb{T}$ and let $A_{\mathcal{B}}=\mathcal{B} * \Xi_{\mathcal{B}}$. Then $A_{\mathcal{B}}$ is a homogeneous Banach space on $\mathbb{T}$ with $\mathcal{W} \subseteq A_{\mathcal{B}} \subseteq \mathcal{C}$ and $(\mathcal{B}, \mathcal{B})=A_{\mathcal{B}}^{*}$

Proof: For $f \in \mathcal{B}$ and $\varphi \in \Xi_{\mathcal{B}}$, we have

$(f * \varphi)(s)=\lim _{N \rightarrow \infty} \sum_{n=-N}^{N}\left(1-\frac{|n|}{N+1}\right) \widehat{f}(n) \widehat{\varphi}(n) e^{i n s}=\left\langle R_{-s} f, \varphi\right\rangle \quad(s \in \mathbb{T})$,

so $A_{\mathcal{B}}$ is a homogeneous Banach space on $\mathbb{T}$ with $A_{\mathcal{B}} \subseteq \mathcal{C}$. For $n \in \mathbb{Z}$ and $\varepsilon>0$, choose $f \in \mathcal{B}, f \neq 0$ such that

$$
|\widehat{f}(n)|=\left|\left\langle f, \alpha^{n}\right\rangle\right| \geq(1-\varepsilon)\|f\|_{\mathcal{B}} \cdot\left\|\alpha^{n}\right\|_{\mathcal{B}^{*}} .
$$

Since $f * \alpha^{n}=\widehat{f}(n) \alpha^{n}$, we thus have

$$
\left\|\alpha^{n}\right\|_{A_{\mathcal{B}}} \leq \frac{\|f\|_{\mathcal{B}} \cdot\left\|\alpha^{n}\right\|_{\mathcal{B}^{*}}}{|\widehat{f}(n)|} \leq \frac{1}{1-\varepsilon}
$$

Hence $\left\|\alpha^{n}\right\|_{A_{\mathcal{B}}}=1$, so $\mathcal{W} \subseteq A_{\mathcal{B}}$. The last statement is just a reformulation of Theorem 4.3 and Proposition 4.4.

\section{Examples}

In this section, we shall determine $\Xi_{\mathcal{B}}^{*}$ for some examples of homogeneous Banach spaces $\mathcal{B}$ on $\mathbb{T}$. We have already seen that $\Xi_{L^{1}}^{*}=\mathcal{M}$ and that $\Xi_{\mathcal{C}}^{*}=L^{\infty}$.

We denote the space of left-continuous functions of bounded variation by $\mathcal{B V C}_{l}$. For $f \in \mathcal{B} \mathcal{V} \mathcal{C}_{l}$, there exists a unique measure $\mu(f)$ with $\widehat{\mu(f)}(0)=0$ and $c \in \mathbb{C}$ such that

$$
f(t)=\mu(f)([0, t))+c \quad(t \in \mathbb{T}) .
$$

Moreover, $f \in \mathcal{B} \mathcal{V} \mathcal{C}_{l}$ is absolutely continuous on $\mathbb{T}$ if and only if $\mu(f) \in$ $L^{1}$, and in this case $f^{\prime}=\mu(f)$ a.e. The space of absolutely continuous functions on $\mathbb{T}$ is denoted by $\mathcal{A C}$. 
This generalizes to (pre)homogeneous Banach spaces on $\mathbb{T}$. For a homogeneous Banach space $\mathcal{B}$ on $\mathbb{T}$, let $\mathcal{B}^{1}$ be the space of functions $f \in \mathcal{A C}$ for which $\mu(f) \in \mathcal{B}$ and equip $\mathcal{B}^{1}$ with the norm $\|f\|_{\mathcal{B}^{1}}=$ $\left\|f^{\prime}\right\|_{\mathcal{B}}+|f(0)|\left(f \in \mathcal{B}^{1}\right)$. Similarly, for a prehomogeneous Banach space $\mathcal{Y}$ on $\mathbb{T}$, we say that a function $f \in \mathcal{B V C}_{l}$ belongs to $\mathcal{Y}^{1}$ if $\mu(f) \in \mathcal{Y}$, and we norm $\mathcal{Y}^{1}$ by $\|f\|_{\mathcal{Y}^{1}}=\|\mu(f)\|_{\mathcal{Y}}+|f(0)|\left(f \in \mathcal{Y}^{1}\right)$. Then $\mathcal{B}^{1}$ is a homogeneous Banach space on $\mathbb{T}$ and $\mathcal{Y}^{1}$ is a prehomogeneous Banach space on $\mathbb{T}$. The following result is easily proved.

Proposition 5.1. Let $\mathcal{B}$ be a homogeneous Banach space on $\mathbb{T}$. Then

$$
\Xi_{\mathcal{B}^{1}}^{*}=\left(\Xi_{\mathcal{B}}^{*}\right)^{1} \text {. }
$$

It immediately follows that

$$
\Xi_{\mathcal{A C}}^{*}=\Xi_{\left(L^{1}\right)^{1}}^{*}=\left(\Xi_{L^{1}}^{*}\right)^{1}=(\mathcal{M})^{1}=\mathcal{B V C}_{l} .
$$

We now turn to other examples.

Dual spaces. The following result is not surprising, considering that the main motivation for the construction of the space $\Xi_{\mathcal{B}}^{*}$ was to embed $\mathcal{B}$ in a "small" dual space.

Lemma 5.2. Let $\mathcal{B}$ be a homogeneous Banach space on $\mathbb{T}$. Suppose that there is a Banach space $\mathcal{Z}$ such that $\mathcal{Z}^{*}=\mathcal{B}$ and such that $\xi_{n} \in \mathcal{Z}$ for $n \in \mathbb{Z}$. Then $\Xi_{\mathcal{B}}=\mathcal{Z}$ and thus $\Xi_{\mathcal{B}}^{*}=\mathcal{B}$.

Proof: Observe that $\mathcal{Z}$ is a closed subspace of $\mathcal{Z}^{* *}=\mathcal{B}^{*}$. Also,

$$
\Xi_{\mathcal{B}}=\overline{\operatorname{span}\left\{\xi_{n}: n \in \mathbb{Z}\right\}}{ }^{\mathcal{B}^{*}}=\overline{\operatorname{span}\left\{\xi_{n}: n \in \mathbb{Z}\right\}}{ }^{\mathcal{Z}},
$$

so $\Xi_{\mathcal{B}}$ is a closed subspace of $\mathcal{Z}$. If $f \in \mathcal{Z}^{*}=\mathcal{B}$ and $f \perp \Xi_{\mathcal{B}}$, then $\widehat{f}(n)=0$ for $n \in \mathbb{Z}$ and thus $f=0$. It thus follows from the HahnBanach theorem that $\Xi_{\mathcal{B}}=\mathcal{Z}$.

The result applies, in particular, to the spaces $L^{p}$ with $1<p<\infty$ and to the Wiener algebra $\mathcal{W} \sim l^{1}(\mathbb{Z})$.

Lipschitz algebras. For $0<\gamma \leq 1$, let $\Lambda_{\gamma}$ be the Lipschitz algebra of functions $f$ on $\mathbb{T}$ for which there exists a constant $C$ such that

$$
|f(t)-f(s)| \leq C|t-s|^{\gamma}
$$

for $s, t \in \mathbb{T}$. Normed by $\|f\|_{\Lambda_{\gamma}}=\|f\|_{\infty}+\sup \left\{|f(t)-f(s)| \cdot|t-s|^{-\gamma}\right.$ : $t, s \in \mathbb{T}, t \neq s\}$, it is well known that $\Lambda_{\gamma}$ is a Banach algebra. For $0<\gamma<1$, let $\lambda_{\gamma}$ be the closed subalgebra of $\Lambda_{\gamma}$ of functions satisfying

$$
|f(t)-f(s)|=o\left(|t-s|^{\gamma}\right)
$$


uniformly as $|t-s| \rightarrow 0$. Then $\lambda_{\gamma}$ is a homogeneous Banach algebra on $\mathbb{T}([\mathbf{1 6}])$. Moreover, it follows from $[\mathbf{3}]$ that the map $\Psi: \lambda_{\gamma}^{* *} \rightarrow \Lambda_{\gamma}$ defined by

$$
\Psi(F)(t)=\left\langle\delta_{t}, F\right\rangle \quad\left(F \in \lambda_{\gamma}^{* *}, t \in \mathbb{T}\right)
$$

is an isomorphism, where $\delta_{t} \in \lambda_{\gamma}^{*}$ denotes the point evalution functional at $t$.

Now, suppose that $F \in \lambda_{\gamma}^{* *}$ with $F \perp \Xi_{\lambda_{\gamma}}$ and let $m \in \mathbb{Z}$. Then

$$
\widehat{\Psi(F)}(m)=\frac{1}{2 \pi} \int_{\mathbb{T}}\left\langle\delta_{t}, F\right\rangle e^{-i m t} d t=\frac{1}{2 \pi}\left\langle\int_{\mathbb{T}} \delta_{t} e^{-i m t} d t, F\right\rangle,
$$

where $\int_{\mathbb{T}} \delta_{t} e^{-i m t} d t$ exists as a $\mathrm{wk}^{*}$ Bochner integral in $\lambda_{\gamma}^{*}$. Since

$$
\begin{aligned}
\frac{1}{2 \pi}\left\langle\alpha^{n}, \int_{\mathbb{T}} \delta_{t} e^{-i m t} d t\right\rangle & =\frac{1}{2 \pi} \int_{\mathbb{T}}\left\langle\alpha^{n}, \delta_{t}\right\rangle e^{-i m t} d t \\
& =\frac{1}{2 \pi} \int_{\mathbb{T}} e^{i(n-m) t} d t=\left\langle\alpha^{n}, \xi_{m}\right\rangle
\end{aligned}
$$

for $n \in \mathbb{Z}$, we deduce that $(1 / 2 \pi) \int_{\mathbb{T}} \delta_{t} e^{-i m t} d t=\xi_{m}$, so

$$
\widehat{\Psi(F)}(m)=\left\langle\xi_{m}, F\right\rangle=0 \text {. }
$$

Hence $\Psi(F)=0$ and thus $F=0$, so we deduce that $\Xi_{\lambda_{\gamma}}=\lambda_{\gamma}^{*}$ and thus

$$
\Xi_{\lambda_{\gamma}}^{*}=\Lambda_{\gamma} \text {. }
$$

Moreover, $\operatorname{span}\left\{\delta_{t}: t \in \mathbb{T}\right\}$ is dense in $\lambda_{\gamma}^{*}([\mathbf{3}$, Lemma 2.6]), so we deduce that a sequence $\left(f_{n}\right)$ in $\Lambda_{\gamma}\left(=\Xi_{\lambda_{\gamma}}^{*}\right)$ converges wk $^{*}$ to 0 if and only if it is bounded in $\Lambda_{\gamma}$ and converges pointwise to 0 on $\mathbb{T}$.

To complete our discussion of Lipschitz algebras, we mention that

$$
\Xi_{\mathcal{C}^{1}}^{*}=\left(\Xi_{\mathcal{C}}^{*}\right)^{1}=\left(L^{\infty}\right)^{1}=\Lambda_{1}
$$

by Proposition 5.1

The Pisier algebra. Let $\left(\xi_{n}(\omega)\right)$ be a sequence of independent, normal, complex random variables defined on some probability space $\Omega$. For $f \in L^{2}$, consider the Gaussian Fourier series

$$
f_{\omega}(t)=\sum_{n=-\infty}^{\infty} \xi_{n}(\omega) \widehat{f}(n) e^{i n t} \quad(t \in \mathbb{T}, \omega \in \Omega) .
$$

We say that $f \in \mathcal{C}$ almost surely if $f_{\omega} \in \mathcal{C}$ for almost every $\omega \in \Omega$. The space $\mathcal{C}_{\text {a.s. }}$ of such functions is a homogeneous Banach space on $\mathbb{T}$ equipped with the norm

$$
\|f\|_{\text {a.s. }}=\int_{\Omega}\left\|f_{\omega}\right\|_{\infty} d \omega \quad\left(f \in \mathcal{C}_{\text {a.s. }}\right) .
$$


Pisier proved that

$$
\mathcal{P}=\mathcal{C}_{\text {a.s. }} \cap \mathcal{C}
$$

is closed under pointwise multiplication and that it is a homogeneous Banach algebra on $\mathbb{T}$ (the so-called Pisier algebra) equipped with the norm

$$
\|f\|_{\mathcal{P}}=\|f\|_{\infty}+\|f\|_{\text {a.s. }} \quad(f \in \mathcal{P}) .
$$

(For this and other results, see, for instance, [17] or [12].)

It follows from $\left[\mathbf{1 5}\right.$, Corollary VI.1.5] that $\mathcal{C}_{\text {a.s. }}=\mathcal{Z}^{*}$ for some Banach space $\mathcal{Z}$ with $\xi_{n} \in \mathcal{Z}$ for $n \in \mathbb{N}$. Hence

$$
\Xi_{\mathcal{P}}^{*} \subseteq \Xi_{\mathcal{C}_{\text {a.s. }}}^{*} \cap \Xi_{\mathcal{C}}^{*}=\mathcal{C}_{\text {a.s. }} \cap L^{\infty}
$$

by Proposition 2.4 and Lemma 5.2. Conversely, for $f \in \mathcal{C}_{\text {a.s. }} \cap L^{\infty}$, the sequence $\left(\sigma_{N}(f)\right)$ is bounded in $\mathcal{C}_{\text {a.s. }}$ and $\mathcal{C}$, so it follows from Corollary 2.9 that $f \in \Xi_{\mathcal{P}}^{*}$. Hence

$$
\Xi_{\mathcal{P}}^{*}=\mathcal{C}_{\text {a.s. }} \cap L^{\infty} \text {. }
$$

Finally, with an obvious notation, we have $\mathcal{C}_{\text {a.s. }}=L_{\text {a.s. }}^{\infty}([\mathbf{1 2}$, p. 58$])$, so we obtain the more symmetric expresion

$$
\Xi_{\mathcal{C}_{\mathrm{a} . \mathrm{s} .}^{*} \cap \mathcal{C}}^{*}=L_{\mathrm{a} . \mathrm{s} .}^{\infty} \cap L^{\infty} .
$$

Uniformly convergent Fourier series. For $\mu \in \mathcal{M}$ and $N \in \mathbb{N}$, let

$$
S_{N}(\mu)=\sum_{n=-N}^{N} \widehat{\mu}(n) \alpha^{n}
$$

be the partial sums of $\mu$, and let

$$
\mathcal{U}=\left\{f \in \mathcal{C}:\left\|S_{N}(f)-f\right\|_{\infty} \rightarrow 0 \text { as } N \rightarrow \infty\right\}
$$

be the space of uniformly convergent Fourier series on $\mathbb{T}$ equipped with the norm

$$
\|f\|_{\mathcal{U}}=\sup _{N}\left\|S_{N}(f)\right\|_{\infty} \quad(f \in \mathcal{U}) .
$$

It is easily seen that $\mathcal{U}$ is a homogeneous Banach space on $\mathbb{T}$. For $\mu \in \Xi_{\mathcal{U}}^{*}$, we have $\widehat{\sigma_{M}(\mu)}(n) \rightarrow \widehat{\mu}(n)$ as $M \rightarrow \infty$ for $n \in \mathbb{Z}$ and thus $\| S_{N}\left(\sigma_{M}(\mu)\right)-$ $S_{N}(\mu) \|_{\infty} \rightarrow 0$ as $M \rightarrow \infty$ for $N \in \mathbb{N}$. Hence

$$
\sup _{N}\left\|S_{N}(\mu)\right\|_{\infty} \leq \sup _{M, N}\left\|S_{N}\left(\sigma_{M}(\mu)\right)\right\|_{\infty}=\sup _{M}\left\|\sigma_{M}(\mu)\right\|_{\mathcal{U}}<\infty
$$


In particular, $\mu \in L^{\infty}$. Conversely, let $f \in L^{\infty}$ with $\sup _{N}\left\|S_{N}(f)\right\|_{\infty}<$ $\infty$. Then $\left(S_{N}(f)\right)$ is bounded in $\mathcal{U}$ and $\widehat{S_{N}(f)}(m) \rightarrow \widehat{f}(m)$ as $N \rightarrow \infty$ for $m \in \mathbb{Z}$. Hence $S_{N}(f) \rightarrow f$ wk $^{*}$ in $\Xi_{\mathcal{U}}^{*}$ as $N \rightarrow \infty$, so we deduce that

$$
\Xi_{\mathcal{U}}^{*}=\left\{f \in L^{\infty}: \sup _{N}\left\|S_{N}(f)\right\|_{\infty}<\infty\right\},
$$

the space of uniformly bounded Fourier series on $\mathbb{T}$.

Spaces of analytic functions. As mentioned earlier, the definition of a homogeneous Banach space $\mathcal{B}$ includes the case where $\widehat{f}(n)=0$ for every $f \in \mathcal{B}$ for some values of $n$ and in particular spaces of analytic functions. For instance, the Hardy space $\mathcal{H}^{1}$ on the open unit disc $\mathbb{D}$ is a homogeneous Banach space on $\mathbb{T}$. Moreover, by the F. and M. Riesz theorem $([\mathbf{1 1}$, p. 47]), we have

$$
\Xi_{\mathcal{H}^{1}}^{*} \subseteq\{\mu \in \mathcal{M}: \widehat{\mu}(n)=0 \text { for every } n<0\}=\mathcal{H}^{1},
$$

so

$$
\Xi_{\mathcal{H}^{1}}^{*}=\mathcal{H}^{1} .
$$

This also follows from Lemma 5.2 , since $\mathcal{H}^{1}=\left(\mathcal{C} / \mathcal{A}_{0}\right)^{*}$, where $\mathcal{A}$ is the disc algebra of functions analytic on $\mathbb{D}$ and continuous on $\overline{\mathbb{D}}$ and $\mathcal{A}_{0}=\{f \in \mathcal{A}: f(0)=0\}$. For the disc algebra, we have $\mathcal{A}^{*}=\mathcal{M} / \mathcal{A}^{\perp}=$ $\mathcal{M} / \mathcal{H}_{0}^{1}$ (where $\mathcal{H}_{0}^{1}=\left\{f \in \mathcal{H}^{1}: f(0)=0\right\}$ ). Hence $\Xi_{\mathcal{A}}=L^{1} / \mathcal{H}_{0}^{1}$ and thus

$$
\Xi_{\mathcal{A}}^{*}=\mathcal{H}^{\infty} \text {. }
$$

Finally, we consider the space

$$
\mathcal{B M O} \mathcal{A}=\mathcal{B M O} \cap \mathcal{H}^{1}
$$

of functions in $\mathcal{H}^{1}$ which are of bounded mean oscillation on $\mathbb{T}$, and the closed subspace $\mathcal{V M O} \mathcal{A}$ of functions of vanishing mean oscillation on $\mathbb{T}$ (see, for example, $[\mathbf{8}]$ for the definitions). Fefferman's duality theorem ([1, Corollary 8.1$]$ or $[\mathbf{8}$, Theorem VI.4.4]) states that

$$
\mathcal{B M O} \mathcal{A}=\left(\mathcal{H}^{1}\right)^{*}
$$

Hence conditions (i) and (iii) in the definition of a prehomogeneous Banach space on $\mathbb{T}$ are satisfied for $\mathcal{B M O} \mathcal{A}$. Moreover, for $g \in \mathcal{B M O A}$, we have $g_{s} \rightarrow g$ wk $^{*}$ in $\mathcal{B M O A}$ as $s \rightarrow 0$, so it follows from the proof of Theorem 2.6 that

$$
f * g=\frac{1}{2 \pi} \int_{\mathbb{T}} f(s) g_{s} d s
$$

exists as a $\mathrm{wk}^{*}$ Bochner integral in $\mathcal{B M O} \mathcal{A}$ for $f \in L^{1}$. Hence

$$
\|f * g\|_{\mathcal{B M O A}} \leq\|f\|_{L^{1}}\|g\|_{\mathcal{B M O A}}
$$


so we deduce that $\mathcal{B M O A}$ is a prehomogeneous Banach space on $\mathbb{T}$. Also,

$$
(\mathcal{B M O A})_{h}=\mathcal{V} \mathcal{M O A}
$$

by $[\mathbf{8}$, Theorem VI.5.1], so it follows from Theorem 2.10 that $\mathcal{V M O} \mathcal{A}$ is a homogeneous Banach space on $\mathbb{T}$ with

$$
\Xi_{\mathcal{V M O A}}^{*}=\mathcal{B M O A} \text {. }
$$

\section{References}

[1] A. Baernstein II, Analytic functions of bounded mean oscillation, in "Aspects of contemporary complex analysis", (D. A. Brannan and J. G. Clunie, eds.), Proceedings of a conference held in Durham, 1979, Academic Press, London, 1980, pp. 3-36.

[2] J. B. Conway, "A course in functional analysis", Springer-Verlag, Berlin, 1985.

[3] K. DE LeEuw, Banach spaces of Lipschitz functions, Studia Math. 21 (1961), 55-66.

[4] N. Dunford and J. T. Schwartz, "Linear operators III", John Wiley \& Sons, New York, 1971.

[5] R. E. Edwards, "Fourier series II", Holt, Rinehart and Winston, New York, 1967.

[6] A. Figà-Talamanca, Translation invariant operators in $L^{p}$, Duke Math. J. 32 (1965), 495-501.

[7] A. Figà-Talamanca and G. I. Gaudry, Density and representation theorems for multipliers of type $(p, q), J$. Austral. Math. Soc. 7 (1967), 1-6.

[8] J. B. Garnett, "Bounded analytic functions", Academic Press, New York, 1981.

[9] E. Hewitt and K. A. Ross, "Abstract harmonic analysis I", Die Grundlehren der mathematischen Wissenschaften 115, SpringerVerlag, Berlin, 1963.

[10] E. Hille AND R. S. Phillips, "Functional analysis and semigroups", revised ed., American Mathematical Society Colloquium Publications, vol. 31, American Mathematical Society, Providence, R.I., 1957.

[11] K. Hoffman, "Banach spaces of analytic functions", Prentice-Hall, Englewood Cliffs, N.J., 1962.

[12] J.-P. Kahane, "Some random series of functions", second ed., Cambridge Studies in Advanced Mathematics 5, Cambridge University Press, Cambridge-New York, 1985. 
[13] Y. Katznelson, "An introduction to harmonic analysis", John Wiley \& Sons, New York, 1968.

[14] R. LARSEN, "The multiplier problem", Lecture Notes in Math. 105, Springer-Verlag, Berlin, 1965.

[15] M. B. Marcus And G. Pisier, "Random Fourier series with applications to harmonic analysis", Annals in Mathematics Studies 101, Princeton University Press, Princeton, N.J., 1981.

[16] H. Mirkil, Continuous translation of Hölder and Lipschitz functions, Canad. J. Math. 12 (1960), 674-685.

[17] G. Pisier, A remarkable homogeneous Banach algebra, Israel J. Math. 34 (1979), 38-44.

[18] M. A. Rieffel, Multipliers and tensor products of $L^{p}$-spaces of locally compact groups, Studia Math. 33 (1969), 71-82.

[19] W. Rudin, Invariant measures on $L^{\infty}$, Studia Math. 44 (1972), 219-227.

[20] G. E. Shilov, Homogeneous rings of functions, Amer. Math. Soc. Transl. (1) 8 (1962), 392-455; reprinted from Amer. Math. Soc. Transl. 92 (1953).

Laboratoire de Mathématiques Pures

Université Bordeaux 1

351, cours de la Libération

F-33405 Talence cédex

France

E-mail address: vils@math.u-bordeaux.fr

Primera versió rebuda el 21 de gener de 1999, darrera versió rebuda el 17 de juny de 1999. 